\title{
Clinical Features and Visual Outcomes of Optic Neuritis in Chinese Children
}

\author{
Huanfen Zhou, ${ }^{1,2}$ Wei Wang, ${ }^{3}$ Quangang $X u,{ }^{2,4}$ Shaoying Tan, \\ Shuo Zhao, ${ }^{5}$ Mo Yang, ${ }^{2}$ Chunxia Peng, ${ }^{2}$ and Shihui Wei ${ }^{2}$ \\ ${ }^{1}$ Department of Ophthalmology, The First Affiliated Hospital of Chinese People's Liberation Army General Hospital, Beijing, China \\ ${ }^{2}$ Department of Ophthalmology, Chinese PLA General Hospital, Beijing, China \\ ${ }^{3}$ Zhongshan Ophthalmic Center, State Key Laboratory of Ophthalmology, Sun Yat-sen University, Guangzhou, China \\ ${ }^{4}$ Department of Neurology, Chinese PLA General Hospital, Beijing, China \\ ${ }^{5}$ Department of Ophthalmology, Beijing Hospital, Beijing, China
}

Correspondence should be addressed to Shihui Wei; docwsh301@126.com

Received 28 April 2016; Revised 2 August 2016; Accepted 25 August 2016

Academic Editor: Terri L. Young

Copyright (C) 2016 Huanfen Zhou et al. This is an open access article distributed under the Creative Commons Attribution License, which permits unrestricted use, distribution, and reproduction in any medium, provided the original work is properly cited.

\begin{abstract}
Purpose. Although optic neuritis $(\mathrm{ON})$ in children is relatively common, visual outcomes and factors associated with the condition have not been well documented. The aim of this study was to evaluate the clinical features and visual outcomes of ON in Chinese children. Methods. Patients with a first episode of $\mathrm{ON}$ at a tertiary neuroophthalmic centre in China were assessed and followed up for at least three months. Visual outcomes and clinical, laboratory, and neuroimaging findings were reviewed. In patients with bilateral ON, only the eyes with worse visual acuity (VA) at presentation were used for statistical analysis. Results. Seventy-six children (76 eyes) with a first episode of $\mathrm{ON}$ were included. The mean age was 11.8 years, $60.5 \%$ were females, and $48.7 \%$ had bilateral involvement. The children were followed up for an average of 18.5 months (age range, 3-48 months). Vision loss at presentation was severe, with VA < 20/200 in 37 eyes (48.7\%). At the final visit, 3 (3.9\%) eyes had VA of at least 20/20, and 41 (53.9\%) eyes had VA of at least 20/40. The final VA in 35 eyes (46.1\%) was worse than 20/40. Children aged $\leq 10$ years had better predicted visual outcomes when compared to children over 10 years (odds ratio $=2.73,95 \%$ confidential interval: $1.05-7.07$, and $P=0.039$ ). The other features of this cohort, such as sex, experienced bilateral attack, VA at presentation, presence of optic disc edema, systemic diseases, magnetic resonance imaging (MRI) findings, and aquaporin-4 (AQP-4) antibody status, were not significantly correlated with the final visual outcome. Conclusion. The data revealed the clinical characteristics and visual outcomes of ON in Chinese children. ON in children was associated with severe vision loss and relatively good visual recovery. The age at onset could predict the final visual function.
\end{abstract}

\section{Introduction}

Optic neuritis $(\mathrm{ON})$ is an inflammatory process of the optic nerve, which may be isolated or closely correlated with systemic demyelinating diseases such as multiple sclerosis (MS) and neuromyelitis optica spectrum disorders (NMOSDs) [1, 2]. Aquaporin-4 (AQP-4) antibody testing has been added to the diagnostic assessment for $\mathrm{ON}$ and has prognostic value $[3,4]$. The prevalence of $\mathrm{ON}$ in children is lower than that in adults. Furthermore, $\mathrm{ON}$ in children is distinguished by greater bilateral involvement, higher prevalence of optic disc edema (ODE), and lower conversion into MS [5]. In the optic neuritis treatment trial (ONTT), $50 \%$ of adults with ON, $72 \%$ patients with one or more lesions in brain MRI, and $25 \%$ of patients without lesion in brain MRI progressed to MS during the 15-year follow-up [6,7]. With regard to children with ON, a meta-analysis demonstrated that only $19 \%$ of the children developed MS after an average follow-up of 6.3 years [8].

The influence of race/ethnicity on features and outcomes of adults with ON has been well documented. In ONTT, people of African American origin were found to have lower visual acuity (VA) and contrast sensitivity at onset and faster vision recovery but worse final visual outcomes in comparison with white patients with ON [9]. Epidemiological 
studies also showed that African Americans had more severe vision loss, worse disability scores, more retinal atrophy, and accelerated retinal nerve fibre-layer thinning compared to Caucasian Americans [10-12]. Furthermore, MS is rare in East Asian populations such as Chinese, Korean, and Japanese populations, and the prognosis of $\mathrm{ON}$ in these populations differs from that in American and European populations. We previously demonstrated a higher prevalence of serum AQP-4 antibody, severe vision loss, and poor vision recovery in Chinese adults with ON, with only $26.1 \%$ of patients having VA of $20 / 20$ or better and $39.1 \%$ of patients having VA of $20 / 100$ or worse at the final visit $[13,14]$.

Although the visual outcomes of adults with $\mathrm{ON}$ are well documented in the ONTT, there are few trials on the outcomes for children with ON. In 2014, Wan et al. reviewed 46 American children (46 eyes) with a first episode of $\mathrm{ON}$ and reported the vision in 35 of 36 eyes (97\%) recovered to $>20 / 40$ after one year [15]. However, most of the children in their study were white. Studies on other races are urgently needed. Therefore, to fill the gap, we performed a retrospective study to investigate the clinical features and visual outcomes of $\mathrm{ON}$ in Chinese children.

\section{Methods}

This was a retrospective observational cohort study on hospitalized patients diagnosed with $\mathrm{ON}$ in the neuroophthalmology department of Chinese People's Liberation Army General Hospital (PLAGH). Patients were recruited between January 2012 and December 2014. The study protocol was approved by the institutional review board at the Chinese PLAGH and performed in accord with the tenets of the Helsinki Declaration. Informed consent was obtained from all the patients or their parents.

2.1. Patients. Patients who presented with a first episode of $\mathrm{ON}$ were identified by searching electronic medical records. The diagnosis of $\mathrm{ON}$ was confirmed by both an experienced neurologist (QX) and ophthalmologist (SW). Patients included in this study had to fulfil the following criteria: (1) the first episode of $\mathrm{ON}$ with best corrected visual acuity (BCVA) loss; (2) age < 18 years; (3) followup of $\geq 3$ months; (4) no prior steroid treatment; and (5) objective evidence of visual abnormalities: relative afferent papillary defect (RAPD), visual field defects, and abnormal visual evoke potential (VEP). Patients with any evidence of the following conditions were excluded: (1) uncertainty in the diagnosis; (2) other types of optic neuropathy such as compressive, hereditary, vascular, toxic, traumatic, metabolic, or infiltrative optic neuropathy; (3) other ocular diseases such as amblyopia, uveitis, or glaucoma; (4) a previous episode of ON; and (5) systemic diseases, such as epilepsy and malignancy. Patients diagnosed with MS, NMOSD, acute disseminated encephalomyelitis (ADEM), or systemic lupus erythematosus (SLE) were not excluded.

2.2. Study Outcomes. The following data were extracted from the medical records using a standardized form: age, sex, laterality, ocular symptoms, pupil function test, fundus findings, colour vision test, and laboratory findings including AQP-4 IgG status and cerebral spinal fluid (CSF) analysis, orbit magnetic resonance imaging (MRI) information, treatment, follow-up, best corrected visual acuity (VA) at presentation, and final VA. The serum AQP-4 antibody examination was assessed using the cell-based assay described previously by our group [31]. The VA in all the children was evaluated using the Snellen visual chart, and the data were transformed as the logarithm of the minimum angle of resolution $(\log$ MAR) values [16]. The MRI images were reviewed by the same experienced radiologist. Moreover, MS and NMOSD were diagnosed according to the latest international criteria $[17,32]$. In patients with bilateral $\mathrm{ON}$, only the eyes with worse BCVA at presentation were included in this study.

2.3. Statistical Analyses. Only one eye of each patient was used for statistical analysis. In patients with bilateral $\mathrm{ON}$, only the eyes with worse visual acuity (VA) at presentation were used for statistical analysis to exclude intereye correlations. All statistical analyses were performed using SPSS 20.0 software (IBM Corporation, New York, USA). Fisher's exact test was used to compare categorical variables between groups and determine the predictors of a favourable visual outcome (final VA $\geq 20 / 40$ ). No adjustment for multiple comparisons was made owing to the exploratory nature of the study. In addition, logistic regression analyses were performed to calculate the odds ratio of poor visual outcome as a function of age onset, sex, laterality, VA at presentation, presence of ODE, systemic diseases, MRI findings, and AQP-4 IgG status. A value of $P<0.05$ was considered statistically significant.

\section{Results}

3.1. Demographic and Clinical Characteristics. Seventy-six children were eligible for this study, with 30 male and 46 female children. Table 1 summarized the demographic and clinical characteristics of these patients (unit of presentation $=$ person). $39(51.3 \%)$ patients experienced unilateral involvement and 37 patients (48.7\%) experienced bilateral involvement. The mean age at onset was 11.8 years (5-17 years). The follow-up duration ranged from 3 to 48 months, with a mean of 18.5 months (median of 11 months). Eighteen patients who experienced bilateral ON had RAPD. Of the 47 patients who underwent AQP-4 IgG testing, 13 (27.7\%) were seropositive and $34(72.3 \%)$ were seronegative. Of the 42 patients who underwent orbit MRI imaging, 35 (83.33\%) had optic nerve T2 lesions and 7 (16.67\%) had normal MRI findings. One patient was diagnosed with ADEM, one patient was diagnosed with SLE, three patients were diagnosed with MS, and 13 patients were diagnosed with neuromyelitis optica spectrum disorders (NMOSDs) during the follow-up. ON was the first symptom in all children and no prior myelitis attacked in children with seropositive AQP4 antibody. During the follow-up, none of the children with AQP-4 Ab seropositive underwent attack of myelitis. 
TABle 1: Demographic and clinical characteristics of Chinese children with optic neuritis.

\begin{tabular}{|c|c|c|}
\hline Features & Total & Percent (\%) \\
\hline Number of patients & 76 & $100 \%$ \\
\hline \multicolumn{3}{|l|}{ Age at onset, year } \\
\hline Mean & $11.80 \pm 3.87$ & \\
\hline Range & 5-17 & \\
\hline \multicolumn{3}{|l|}{ Sex } \\
\hline Male & 30 & 39.5 \\
\hline Female & 46 & 60.5 \\
\hline Experience & 76 & \\
\hline Unilateral attack & 39 & 51.3 \\
\hline Bilateral attack & 37 & 48.7 \\
\hline Pupillary function test & 76 & \\
\hline RAPD positive in unilateral & 39 & 100 \\
\hline RAPD positive in bilateral & 18 & 48.65 \\
\hline AQP-4 IgG status & 47 & \\
\hline Seropositive & 13 & 27.7 \\
\hline Seronegative & 34 & 72.3 \\
\hline Orbit MRI & 42 & \\
\hline Abnormal ( $\mathrm{T}_{2}$ lesions) & 35 & 83.33 \\
\hline Normal & 7 & 16.67 \\
\hline Causes/associations & 76 & \\
\hline ION & 58 & 76.3 \\
\hline ADEM & 1 & 1.3 \\
\hline MS & 3 & 4.0 \\
\hline SLE & 1 & 1.3 \\
\hline NMOSD & 13 & 17.1 \\
\hline Funduscopy* & 76 & \\
\hline Optic disc edema & 22 & 28.9 \\
\hline Normal & 54 & 71.1 \\
\hline Colour vision test ${ }^{*}$ & 55 & \\
\hline Deficit & 11 & 20 \\
\hline Intact & 33 & 60 \\
\hline Vision too poor to test & 11 & 20 \\
\hline Presenting VA* & 76 & \\
\hline $20 / 40 \leq \mathrm{VA}<20 / 20$ & 5 & 6.6 \\
\hline $20 / 200 \leq \mathrm{VA}<20 / 40$ & 34 & 44.7 \\
\hline $\mathrm{CF} \leq \mathrm{VA}<20 / 200$ & 6 & 7.9 \\
\hline $\mathrm{NLP} \leq \mathrm{VA}<\mathrm{CF}$ & 31 & 40.8 \\
\hline \multicolumn{3}{|l|}{ Treatment } \\
\hline IV methylprednisolone & 76 & 100 \\
\hline
\end{tabular}

RAPD, relative afferent papillary defect; AQP-4 IgG, anti-aquaporin-4 antibody IgG; ION, isolated optic neuritis; ADEM, acute disseminated encephalomyelitis; MS, multiple sclerosis; SLE, systemic lupus erythematosus; IV, intravenous; VA, best corrected visual acuity; CF, count finger; and NLP, no light perception.

* Only one eye of each patient was included in the statistical analyses.

To eliminate intereye correlations, data of only one eye in each patient was included in the statistical analysis (unit of analysis $=$ eye). Twenty-two $(28.9 \%)$ eyes had ODE. Of 55 eyes with colour vision test, $11(22 \%)$ eyes had colour vision deficit. Vision loss at presentation was severe, with
VA $<20 / 200$ in $37(48.7 \%)$ eyes. All patients received intravenous methylprednisolone therapy (10-30 mg/kg/day) for three days, followed by oral prednisolone $(1 \mathrm{mg} / \mathrm{kg} /$ day for two weeks).

3.2. Visual Outcomes. VA was determined in the affected eyes in unilateral $\mathrm{ON}$ and in the worse eyes at presentation in bilateral ON. The VA at presentation of the patients aged $\leq 10$ years was not significantly different from that of the patients aged $>10$ years (Table 2). 13 (43.3\%) patients aged $\leq 10$ years and $24(52.2 \%)$ patients aged $>10$ years had VA $<20 / 200$. At the final visit, among those aged $\leq 10$ years, the VA of 21 ( $70 \%)$ patients had improved to at least $20 / 40$, as compared to that of $20(43.5 \%)$ patients aged $<10$ years.

3.3. Predictors of Visual Outcomes. The age at onset was significantly correlated with the final VA. The children aged $\leq$ 10 years had better predicted visual outcomes when compared to those older than 10 years (odds ratio $=2.73,95 \%$ confidential interval: 1.05-7.07, and $P=0.039$ ). The other features of this cohort, such as sex, experienced bilateral attack, VA at presentation, presence of ODE, systemic diseases, MRI findings, and AQP-4 IgG status, were not significantly correlated with the final visual outcomes (Table 3).

\section{Discussion}

In this study, we described the clinical features and visual outcomes of $\mathrm{ON}$ in Chinese children. The clinical features of this cohort included female preponderance (60.5\%), common bilateral involvement $(48.7 \%)$, and a high proportion of ODE (28.9\%), which is consistent with previous reports of ON in various ethnic groups (Table 4) [15, 18-30, 33-36]. The vision loss was severe with $48.7 \%$ of patients having VA $<20 / 200$. However, the visual recovery was favourable in the majority of children, with VA at least $20 / 40$ in $53.9 \%$ of the patients. The only identified predictor of visual outcomes was the age at onset. To the best of our knowledge, this is the first study to report the characteristics of ON in children in Mainland China and represents the largest cohort of children with $\mathrm{ON}$.

Few studies have reported the visual outcomes of $\mathrm{ON}$ in children to date. Brady et al. examined 25 children (39 eyes) with $\mathrm{ON}$ and reported that 30 of $39(76 \%)$ eyes recovered to 20/40 or better after a follow-up of an average of 11 months [30]. Wilejto et al. reviewed 36 children (51 eyes) with ON in Canada and reported that the VA of 39 of 47 eyes (83\%) recovered to $\geq 20 / 40$ after a follow-up of 2.4 years [26]. In Japanese children with ON, Mizota et al. reported that 54 of 61 (95\%) eyes recovered to $20 / 20$ or better after a mean followup of 10.7 years [27]. A study of Korean children with ON reported that the VA recovered to $\geq 20 / 40$ in $53.3 \%-80 \%$ of affected eyes but that it remained $\leq 20 / 200$ in $7.7 \%-13.3 \%$ of eyes at the final visit $[18,22,25,28]$. A study of Malaysian children revealed that the final VA of 21 of 28 (75\%) eyes was $\geq 20 / 40$ [21]. Among children with ON in Taiwan, Sun et al. reported that the VA of 20 of $24(83.3 \%)$ eyes recovered to $\geq 20 / 40$ after a mean follow-up of 14.01 months [23]. In the 
TABLE 2: Visual outcomes of Chinese children with optic neuritis.

\begin{tabular}{|c|c|c|c|c|}
\hline \multirow{2}{*}{ Visual outcomes (worse eye at onset if bilateral) } & \multicolumn{2}{|c|}{ Age $\leq 10$ years } & \multicolumn{2}{|c|}{ Age $>10$ years } \\
\hline & Initial & Final & Initial & Final \\
\hline $\mathrm{VA} \geq 20 / 20$ & 0 & $3(10 \%)$ & 0 & 0 \\
\hline $20 / 40 \leq \mathrm{VA}<20 / 20$ & $3(10 \%)$ & $18(60 \%)$ & $2(4.4 \%)$ & $20(43.5 \%)$ \\
\hline $20 / 200 \leq \mathrm{VA}<20 / 40$ & $14(46.7 \%)$ & $7(23.3 \%)$ & $20(43.5 \%)$ & $22(47.8 \%)$ \\
\hline $\mathrm{CF} \leq \mathrm{VA}<20 / 200$ & $1(3.3 \%)$ & 0 & $5(10.9 \%)$ & 0 \\
\hline $\mathrm{NLP} \leq \mathrm{VA}<\mathrm{CF}$ & $12(40 \%)$ & $2(6.7 \%)$ & $19(41.3 \%)$ & $4(8.7 \%)$ \\
\hline Total & 30 & 30 & 46 & 46 \\
\hline
\end{tabular}

VA, visual acuity; CF, count finger; and NLP, no light perception.

TABLE 3: Factors for visual outcomes in Chinese children with optic neuritis.

\begin{tabular}{|c|c|c|c|c|}
\hline Factors & & Total eyes & Final VA $\geq 20 / 40(\%)$ & $P$ value \\
\hline \multirow{2}{*}{ Age at onset } & Age $\leq 10$ years & 30 & $21(70 \%)$ & \multirow{2}{*}{0.039} \\
\hline & Age $>10$ years & 46 & $20(43.5 \%)$ & \\
\hline \multirow{2}{*}{ Sex } & Female & 46 & $24(52.17 \%)$ & \multirow{2}{*}{0.701} \\
\hline & Male & 30 & $17(56.7 \%)$ & \\
\hline \multirow{2}{*}{ Experience } & Unilateral attack & 39 & $20(51.3 \%)$ & \multirow{2}{*}{0.632} \\
\hline & Bilateral attack & 37 & $21(56.8 \%)$ & \\
\hline \multirow{2}{*}{ Initial VA } & $\mathrm{VA}>\mathrm{CF}$ & 42 & $25(59.5 \%)$ & \multirow{2}{*}{0.278} \\
\hline & $\mathrm{VA} \leq \mathrm{CF}$ & 34 & $16(47.1 \%)$ & \\
\hline \multirow{2}{*}{ Funduscopy } & ODE & 22 & $14(63.6 \%)$ & \multirow{2}{*}{0.501} \\
\hline & Normal & 54 & $30(55.6 \%)$ & \\
\hline \multirow{2}{*}{ Systemic diseases } & ADEM/MS/SLE/NMOSD & 18 & $8(44.4 \%)$ & \multirow{2}{*}{0.454} \\
\hline & ION & 58 & $32(55.2 \%)$ & \\
\hline \multirow{2}{*}{ Orbital MRI imaging } & Normal & 7 & $4(57.1 \%)$ & \multirow{2}{*}{0.934} \\
\hline & Abnormal ( $\mathrm{T}_{2}$ lesions) & 35 & $20(58.8 \%)$ & \\
\hline \multirow{2}{*}{ AQP-4 IgG status } & Seropositive & 13 & $5(38.5 \%)$ & \multirow{2}{*}{0.374} \\
\hline & Seronegative & 34 & $18(52.9 \%)$ & \\
\hline
\end{tabular}

VA, visual acuity; CF, count finger; ODE, optic disc edema; ION, isolated optic neuritis; ADEM, acute disseminated encephalomyelitis; MS, multiple sclerosis; SLE, systemic lupus erythematosus; AQP-4 IgG, anti-aquaporin-4 antibody IgG; and MRI, magnetic resonance imaging.

TABLE 4: Clinical features of children optic neuritis in previous published reports.

\begin{tabular}{|c|c|c|c|c|c|c|c|c|c|}
\hline First author & Year & Location & Patient (eyes) & Female & Mean age & Bilateral & ODE & MS & NMOSD \\
\hline Present & & China & $76(76)$ & $60.5 \%$ & 11.8 & $48.7 \%$ & $36.7 \%$ & $3.95 \%$ & $17.1 \%$ \\
\hline Kim [18] & 2015 & Korea & $26(40)$ & $54.0 \%$ & 10.3 & $54.0 \%$ & $77.0 \%$ & $8.0 \%$ & 0 \\
\hline Wan [15] & 2014 & USA & $46(46)$ & $72.0 \%$ & 12.6 & $41.0 \%$ & $67.0 \%$ & $39.0 \%$ & NR \\
\hline Jayakody [19] & 2014 & USA & $26(38)$ & $73.1 \%$ & $4.5-19$ & $46.0 \%$ & $73.0 \%$ & $7.7 \%$ & 0 \\
\hline Shatriah [21] & 2012 & Malaysia & $14(28)$ & $85.7 \%$ & 11.1 & $100 \%$ & $85.8 \%$ & $14.3 \%$ & NR \\
\hline Jo [22] & 2011 & Korea & $20(33)$ & $85.0 \%$ & 6.5 & $65.0 \%$ & $75.8 \%$ & $25.0 \%$ & NR \\
\hline Sri-Udomkajorn [33] & 2011 & Thailand & 31 & $65.0 \%$ & 9.2 & $74.2 \%$ & $55.0 \%$ & $6.0 \%$ & $6.5 \%$ \\
\hline Sun $[23]$ & 2011 & Taiwan & $24(38)$ & $58.3 \%$ & 10.1 & $58.3 \%$ & $63.2 \%$ & $12.5 \%$ & NR \\
\hline Hwang [25] & 2007 & Korea & $10(15)$ & $50.0 \%$ & 7.31 & NR & NR & NR & NR \\
\hline Wilejto [26] & 2006 & Canada & $36(51)$ & $58.0 \%$ & 12.2 & $42.0 \%$ & $67.0 \%$ & $36.0 \%$ & $2.8 \%$ \\
\hline Mizota [27] & 2004 & Japan & $41(61)$ & $56.0 \%$ & 9.4 & $49.0 \%$ & $74.0 \%$ & $31.7 \%$ & NR \\
\hline Hwang [28] & 2002 & Korea & $23(43)$ & $43.0 \%$ & 8.9 & $87.0 \%$ & $51.0 \%$ & $4.0 \%$ & NR \\
\hline Morales [29] & 2000 & USA & 15 & $60.0 \%$ & 9.8 & $66.0 \%$ & $64.0 \%$ & $26.0 \%$ & NR \\
\hline Brady [30] & 1999 & USA & $25(39)$ & $52.0 \%$ & 9.4 & $54.0 \%$ & NR & $16.0 \%$ & NR \\
\hline Visudhiphan [34] & 1995 & Thailand & $22(41)$ & $54.5 \%$ & 7.1 & $86.3 \%$ & $48.7 \%$ & $9.1 \%$ & NR \\
\hline Kriss [35] & 1988 & UK & 39 & $74.0 \%$ & 8.6 & $74.0 \%$ & $74.0 \%$ & $15.0 \%$ & NR \\
\hline Riikonen [36] & 1988 & Finland & 21 & NR & NR & $62.0 \%$ & $76.0 \%$ & $43.0 \%$ & NR \\
\hline
\end{tabular}

ODE, optic disc edema; MS, multiple sclerosis; NMOSD, neuromyelitis optica spectrum disorders; and NR, not reported. 
present study, the VA of $53.9 \%$ of the patients recovered to $\geq 20 / 40$, which is similar to the visual outcomes reported in Korean children [25].

The ONTT reported that $50 \%$ of patients had VA of $20 / 20$ or better and $68 \%$ of patients had VA of $20 / 40$ or better after one year [37]. In American children with ON, Wan et al. reported that $81 \%$ of children had VA of $20 / 20$ or better and $89 \%$ of children had VA of $20 / 40$ or better at the one-year follow-up visit [15]. In this Chinese cohort, we also observed a favourable visual prognosis in the majority of the population, thereby indicating that children with $\mathrm{ON}$ have at least similar or better visual outcomes compared to those in adults.

The identification of predictors of visual outcomes is essential for individualized treatment. The ONTT study demonstrated that race significantly affected the visual prognosis, but it found no associations between age, sex, treatment, and visual outcomes in follow-up periods of 5,10 , or 15 years [6]. In this study, we found that the age at onset was significantly correlated with the visual outcome of the children. Brady et al. examined 25 children (39 eyes) with $\mathrm{ON}$ and reported a trend towards better final VA in patients aged $<6$ years, but this did not have statistical significance [30]. A study in Taiwan reported that poor visual recovery $(<20 / 40)$ in children was associated with younger age $(\leq 10$ years) but not with initial VA, sex, laterality, underlying diagnosis, and abnormalities of brain MRI [23]. However, two other studies found no association between the age at onset and final VA $[15,21]$. The discrepancy may be caused by different ethnicities, small sample size, distribution of ages, various phenotypes of $\mathrm{ON}$, or different follow-up times. Consistent with most previous studies, we confirmed that several baseline parameters such as sex, laterality, initial visual acuity, appearance of ODE, MRI findings, and treatment did not affect the final visual function $[15,19,23,33]$.

Severe vision loss, a high prevalence of AQP-4 IgG, and poor visual recovery were reported to be common in Chinese adults with $\mathrm{ON}$ [38]. We performed AQP-4 IgG testing in 47 patients, and 13 (27.7\%) were seropositive. However, we did not observe any correlations between underlying diagnosis, AQP-4 IgG status, and final VA in this study. The small sample size in our subgroup analysis may explain the lack of correlations. Banwell et al. found that AQP-4 IgG was seropositive in $78 \%$ of children with recurrent NMO [39]. Absoud et al. reviewed 20 children with NMOSD in the UK and reported that the VA of 10 of 12 patients with seropositive AQP-4 IgG was $<20 / 200$ in at least one eye and the bilateral VA was $<20 / 200$ in $58 \%$ of the patients after a mean follow-up of 6.1 years [40]. Most previous studies of ON in children did not detect the serum AQP-4 IgG status. In a Korean study by Kim et al. the status of AQP-4 IgG was only available in three patients [18]. Thus, prospective studies with a large sample size and various ethnicities are required to clarify the influence of AQP-4 IgG on final visual outcomes in children with ON.

The strengths of this study included the uniform sample of children with the first episode of $\mathrm{ON}$, standardized treatment, and detection of AQP-4 IgG status. On the other hand, this study also has a number of limitations. First, the nature of the retrospective design introduces selection bias, precluding the confirmation of the cause and effect relationship. Second, all the patients were derived from a tertiary neuroophthalmic centre in China. Thus, the findings of this study may not be applicable to a larger population or other ethnicities. Third, in the association analyses, there were a relatively small number of patients in several of the subgroups, thereby causing nonsignificance associations between certain factors and visual outcomes. Fourth, we were not able to construct any useful statistics about MS-like lesions or typical NMOSD lesions in brain MRI because only a very small number of patients received the brain MRI or the spine cord MRI. Furthermore, myelin oligodendrocyte glycoprotein (MOG) antibody was not available in our department, which was reliably associated with a spectrum of demyelinating disorders including relapsing paediatric demyelination [41, 42]. Fifth, the follow-up was not standardized and relatively short, and analyses of the conversion rate of MS and NMOSD were not performed. Further, Sun et al. reported a relatively low conversion rate $(12.5 \%)$ of $\mathrm{MS}$ in children with $\mathrm{ON}$ in Taiwan and ADEM was reported to be the most common systemic cause [23]. In Korean children, Kim et al. showed that only two patients (7.7\%) developed MS [18]. More studies with longer-term follow-up and standardized evaluations are necessary.

In summary, the clinical features of $\mathrm{ON}$ in Chinese children were similar to those of children in other Asian countries. Paediatric ON was associated with severe vision loss and relatively good visual recovery. The majority of this cohort (53.9\%) had final VA of at least 20/40. Age at onset of $<10$ years predicted better visual outcomes. No other clinical parameters were significantly correlated with the final visual function.

\section{Competing Interests}

The authors declare that there are no competing interests.

\section{Authors' Contributions}

Huanfen Zhou and Wei Wang contributed equally to this work and share the first authorship. All the authors concur with the submission.

\section{Acknowledgments}

This work was supported by a grant from the National High Technology Research and Development Program of China (863 Program) (no. 2015AAO20511).

\section{References}

[1] A. Langer-Gould, J. L. Zhang, J. Chung, Y. Yeung, E. Waubant, and J. Yao, "Incidence of acquired CNS demyelinating syndromes in a multiethnic cohort of children," Neurology, vol. 77, no. 12, pp. 1143-1148, 2011.

[2] B. Banwell, J. Kennedy, D. Sadovnick et al., "Incidence of acquired demyelination of the CNS in Canadian children," Neurology, vol. 72, no. 3, pp. 232-239, 2009. 
[3] M. Matiello, V. A. Lennon, A. Jacob et al., "NMO-IgG predicts the outcome of recurrent optic neuritis," Neurology, vol. 70, no. 23, pp. 2197-2200, 2008.

[4] S. Jarius, J. Frederikson, P. Waters et al., "Frequency and prognostic impact of antibodies to aquaporin-4 in patients with optic neuritis," Journal of the Neurological Sciences, vol. 298, no. 1-2, pp. 158-162, 2010.

[5] J. E. Collinge and D. T. Sprunger, "Update in pediatric optic neuritis," Current Opinion in Ophthalmology, vol. 24, no. 5, pp. 448-452, 2013.

[6] Optic Neuritis Study G, "Visual function 15 years after optic neuritis: a final follow-up report from the Optic Neuritis Treatment Trial," Ophthalmology, vol. 115, no. 6, pp. 10791082.e5, 2008.

[7] Optic Neuritis Study Group, "Multiple sclerosis risk after optic neuritis: final optic neuritis treatment trial follow-up," Archives of Neurology, vol. 65, no. 6, pp. 727-732, 2008.

[8] A. T. Waldman, L. B. Stull, S. L. Galetta, L. J. Balcer, and G. T. Liu, "Pediatric optic neuritis and risk of multiple sclerosis: meta-analysis of observational studies," Journal of American Association for Pediatric Ophthalmology and Strabismus, vol. 15, no. 5, pp. 441-446, 2011.

[9] H. E. Moss, W. Gao, L. J. Balcer, and C. E. Joslin, "Association of race/ethnicity with visual outcomes following acute optic neuritis: an analysis of the optic neuritis treatment trial," JAMA Ophthalmology, vol. 132, no. 4, pp. 421-427, 2014.

[10] D. J. Kimbrough, E. S. Sotirchos, J. A. Wilson et al., "Retinal damage and vision loss in African American multiple sclerosis patients," Annals of Neurology, vol. 77, no. 2, pp. 228-236, 2015.

[11] J. R. Rinker II, K. Trinkaus, R. T. Naismith, and A. H. Cross, "Higher IgG index found in African Americans versus Caucasians with multiple sclerosis," Neurology, vol. 69, no. 1, pp. 68-72, 2007.

[12] R. A. Marrie, G. Cutter, T. Tyry, T. Vollmer, and D. Campagnolo, "Does multiple sclerosis-associated disability differ between races?” Neurology, vol. 66, no. 8, pp. 1235-1240, 2006.

[13] H. Li, Y. Wang, Q. Xu et al., "Features of anti-aquaporin 4 antibody-seropositive Chinese patients with neuromyelitis optica spectrum optic neuritis," Journal of Neurology, vol. 262, no. 10, pp. 2293-2304, 2015.

[14] H. Li, Y. Zhang, Z. Yi, D. Huang, and S. Wei, "Frequency of autoantibodies and connective tissue diseases in Chinese patients with optic neuritis," PLoS ONE, vol. 9, no. 6, Article ID e99323, 2014.

[15] M. J. Wan, O. Adebona, L. A. Benson, M. P. Gorman, and G. Heidary, "Visual outcomes in pediatric optic neuritis," American Journal of Ophthalmology, vol. 158, no. 3, pp. 503.e2507.e2, 2014.

[16] A. Petzold, M. P. Wattjes, F. Costello et al., "The investigation of acute optic neuritis: a review and proposed protocol," Nature Reviews Neurology, vol. 10, no. 8, pp. 447-458, 2014.

[17] C. H. Polman, S. C. Reingold, B. Banwell et al., "Diagnostic criteria for multiple sclerosis: 2010 revisions to the McDonald criteria," Annals of Neurology, vol. 69, no. 2, pp. 292-302, 2011.

[18] Y. M. Kim, H. Y. Kim, M. J. Cho et al., "Optic neuritis in Korean children: low risk of subsequent multiple sclerosis," Pediatric Neurology, vol. 53, no. 3, pp. 221-225, 2015.

[19] H. Jayakody, D. J. Bonthius, R. Longmuir, and C. Joshi, "Pediatric optic neuritis: does a prolonged course of steroids reduce relapses? A preliminary study," Pediatric Neurology, vol. 51, no. 5, pp. 721-725, 2014.
[20] D. Rappoport, N. Goldenberg-Cohen, J. Luckman, and H. Leiba, "Parainfectious optic neuritis: manifestations in children vs adults," Journal of Neuro-Ophthalmology, vol. 34, no. 2, pp. 122-129, 2014.

[21] I. Shatriah, A.-R. Adlina, S. Alshaarawi, and W.-H. Wan-Hitam, "Clinical profile of Malay children with optic neuritis," Pediatric Neurology, vol. 46, no. 5, pp. 293-297, 2012.

[22] D. H. Jo, S.-J. Kim, J. H. Chae, and Y. S. Yu, "The clinical characteristics of optic neuritis in Korean children," Korean Journal of Ophthalmology, vol. 25, no. 2, pp. 116-120, 2011.

[23] M.-H. Sun, H.-S. Wang, K.-J. Chen et al., "Clinical characteristics of optic neuritis in Taiwanese children," Eye, vol. 25, no. 11, pp. 1457-1464, 2011.

[24] N. Chirapapaisan and M. S. Borchert, "Pediatric optic neuritis," Journal of the Medical Association of Thailand, vol. 91, no. 3, pp. 323-330, 2008.

[25] J. S. Hwang, S. J. Kim, Y. S. Yu, and H. Chung, "Clinical characteristics of multiple sclerosis and associated optic neuritis in Korean children," Journal of AAPOS, vol. 11, pp. 559-563, 2007.

[26] M. Wilejto, M. Shroff, J. R. Buncic, J. Kennedy, C. Goia, and B. Banwell, "The clinical features, MRI findings, and outcome of optic neuritis in children," Neurology, vol. 67, no. 2, pp. 258-262, 2006.

[27] A. Mizota, M. Niimura, and E. Adachi-Usami, "Clinical characteristics of Japanese children with optic neuritis," Pediatric Neurology, vol. 31, no. 1, pp. 42-45, 2004.

[28] J.-M. Hwang, Y. J. Lee, and M. K. Kim, "Optic neuritis in Asian children," Journal of Pediatric Ophthalmology and Strabismus, vol. 39, no. 1, pp. 26-32, 2002.

[29] D. S. Morales, R. M. Siakowski, C. W. Howard, and R. Warman, "Optic neuritis in children," Journal of Ophthalmic Nursing \& Technology, vol. 19, no. 6, pp. 270-276, 2000.

[30] K. M. Brady, A. S. Brar, A. G. Lee, D. K. Coats, E. A. Paysse, and P. G. Steinkuller, "Optic neuritis in children: clinical features and visual outcome," Journal of AAPOS, vol. 3, no. 2, pp. 98-103, 1999.

[31] Y. Yang, D.-H. Huang, W.-P. Wu, L. Wu, L.-F. Chen, and Q. Wu, "The role of aquaporin-4 antibodies in Chinese patients with neuromyelitis optica," Journal of Clinical Neuroscience, vol. 20, no. 1, pp. 94-98, 2013.

[32] D. M. Wingerchuk, B. Banwell, J. L. Bennett et al., "International consensus diagnostic criteria for neuromyelitis optica spectrum disorders," Neurology, vol. 85, no. 2, pp. 177-189, 2015.

[33] S. Sri-Udomkajorn and K. Pongwatcharaporn, "Clinical features and outcome of childhood optic neuritis at Queen Sirikit National Institute of Child Health," Journal of the Medical Association of Thailand, vol. 94, supplement 3, pp. S189-S194, 2011.

[34] P. Visudhiphan, S. Chiemchanya, and S. Santadusit, "Optic neuritis in children: recurrence and subsequent development of multiple sclerosis," Pediatric Neurology, vol. 13, no. 4, pp. 293295, 1995.

[35] A. Kriss, D. A. Francis, F. Cuendet et al., "Recovery after optic neuritis in childhood," Journal of Neurology, Neurosurgery and Psychiatry, vol. 51, no. 10, pp. 1253-1258, 1988.

[36] R. Riikonen, M. Donner, and H. Erkkila, "Optic neuritis in children and its relationship to multiple sclerosis: a clinical study of 21 children," Developmental Medicine and Child Neurology, vol. 30, no. 3, pp. 349-359, 1988. 
[37] M. J. Kupersmith, R. L. Gal, R. W. Beck et al., "Visual function at baseline and 1 month in acute optic neuritis: predictors of visual outcome," Neurology, vol. 69, no. 6, pp. 508-514, 2007.

[38] C. Lai, G. Tian, T. Takahashi, W. Liu, L. Yang, and X. Zhang, "Neuromyelitis optica antibodies in patients with severe optic neuritis in China," Journal of Neuro-Ophthalmology, vol. 31, no. 1, pp. 16-19, 2011.

[39] B. Banwell, S. Tenembaum, V. A. Lennon et al., "Neuromyelitis optica-IgG in childhood inflammatory demyelinating CNS disorders," Neurology, vol. 70, no. 5, pp. 344-352, 2008.

[40] M. Absoud, M. J. Lim, R. Appleton et al., "Paediatric neuromyelitis optica: clinical, MRI of the brain and prognostic features," Journal of Neurology, Neurosurgery and Psychiatry, vol. 86, no. 4, pp. 470-472, 2015.

[41] Y. Hacohen, M. Absoud, K. Deiva et al., "Myelin oligodendrocyte glycoprotein antibodies are associated with a non-MS course in children," Neurology: Neuroimmunology \& Neuroinflammation, vol. 2, no. 2, article e81, 2015.

[42] M. Reindl and K. Rostasy, "MOG antibody-associated diseases," Neurology Neuroimmunology \& Neuroinflammation, vol. 2, article e60, 2015. 


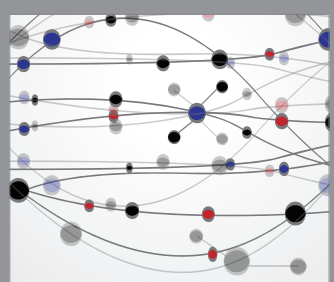

The Scientific World Journal
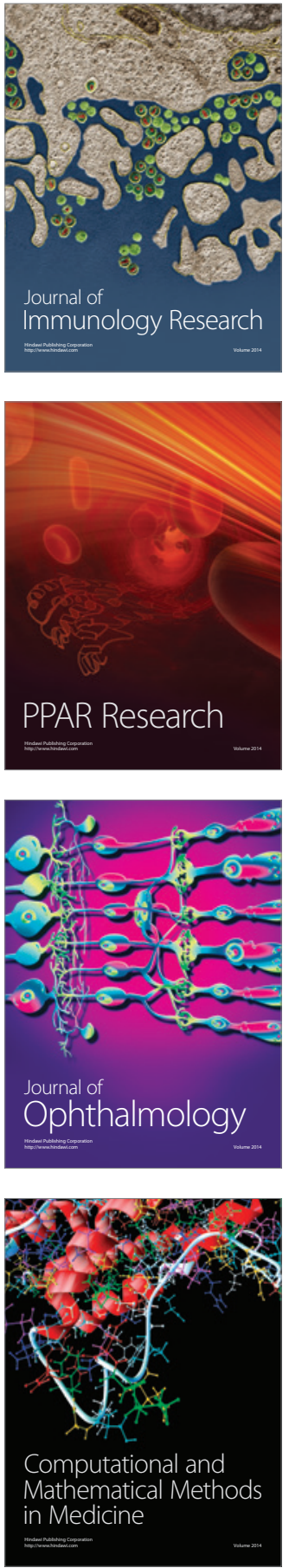

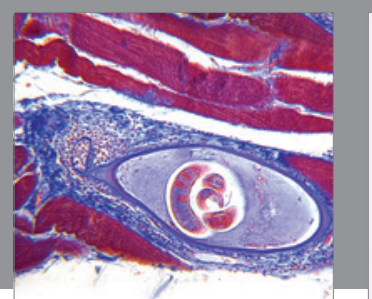

Gastroenterology Research and Practice

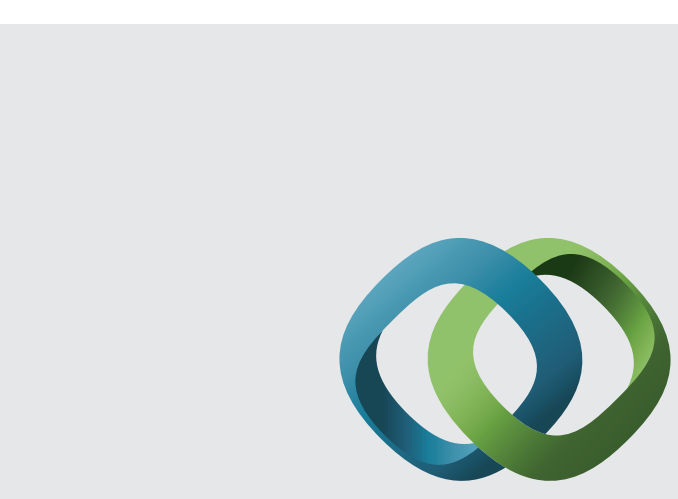

\section{Hindawi}

Submit your manuscripts at

http://www.hindawi.com
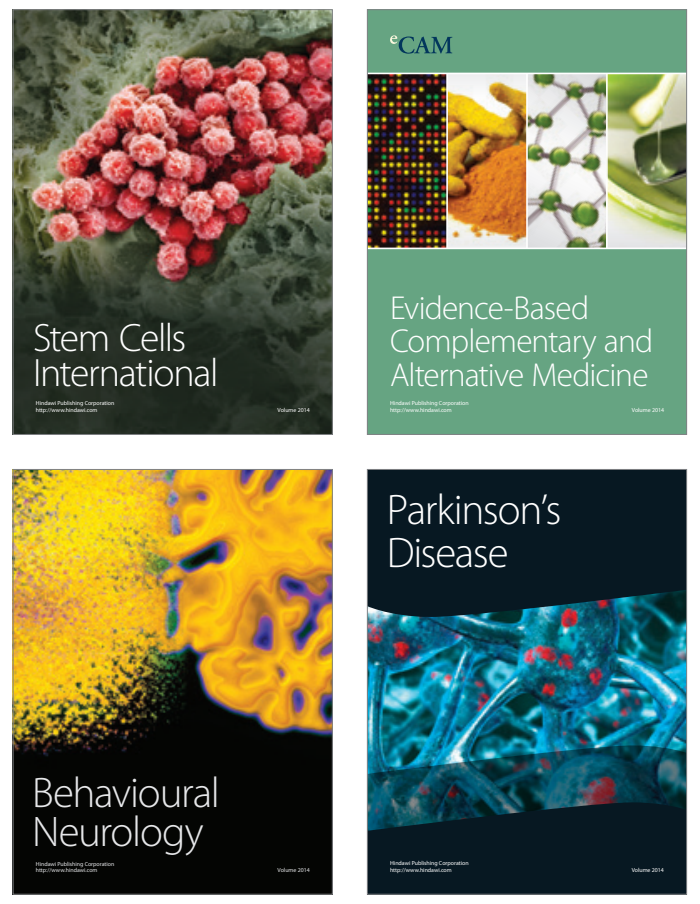
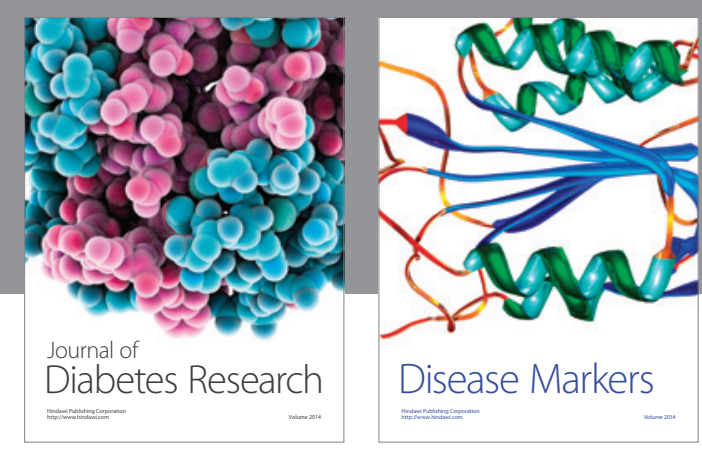

Disease Markers
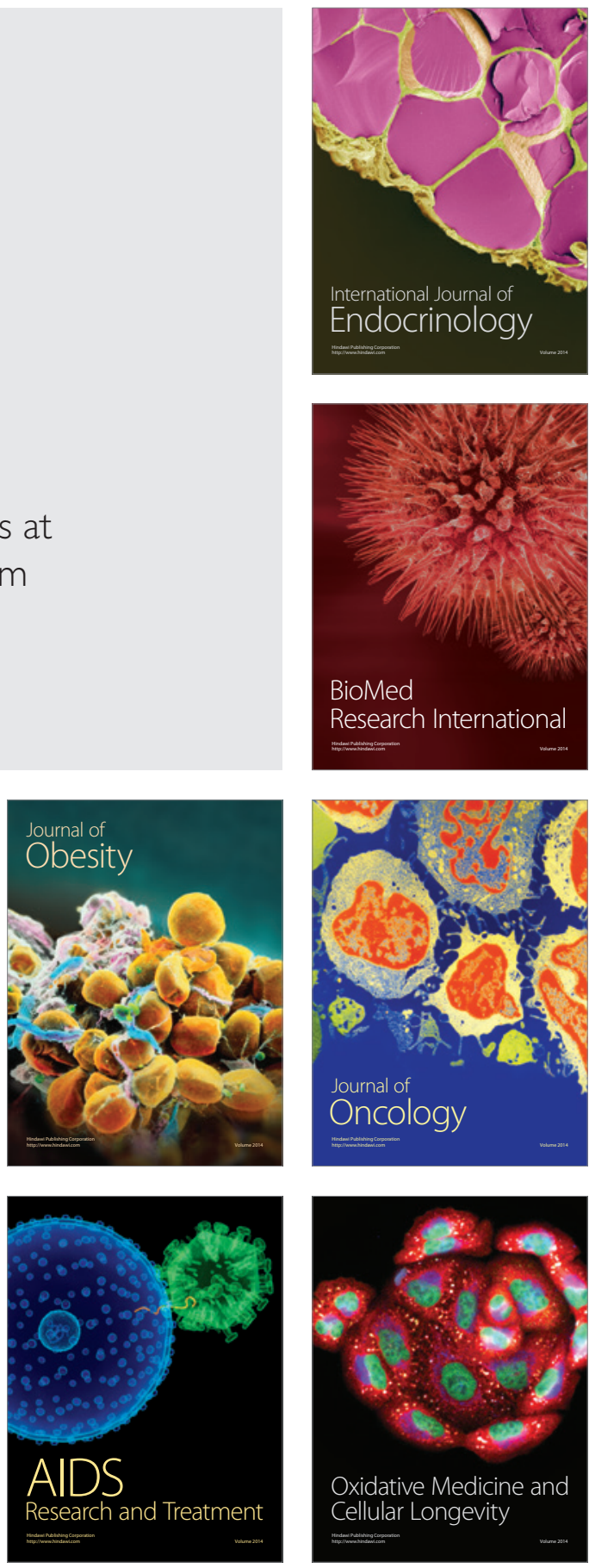